ruhen auf einem breiten Fundament leitfadengestützter Interviews mit Abgeordneten und Funktionsträgern der Fraktionen. Unverkennbar ist, dass der Autor ein exzellenter Kenner des Parlaments aus politikwissenschaftlicher Perspektive und zudem ein ausgewiesener Fachmann des Bundestages ist.

Für die Parlamentskulturforschung liegt ein zentrales Resultat von Helmar Schönes Arbeit darin, „Parlamentskultur als Kombination von Alltagswissen und Alltagshandeln im parlamentarischen Raum zu denken“ (S. 377). Der von Hans-Peter Schwarz herausgegebene Sammelband beleuchtet die Rolle der CDU/CSU-Bundestagsfraktion in der parlamentarischen Entwicklung der Bundesrepublik. Unübersehbar sind an manchen Stellen die Eigenschaften einer Festschrift. Nicht nur die historischen Leistungen der Fraktion werden gewürdigt, sondern auch immer wieder mit wohlmeinenden Elogen auf den Jubilar verbunden. Während die meisten Kapitel eine wissenschaftlich-kritische Distanz vermissen lassen, bietet das Schlusskapitel von Schwarz den größten Gewinn. In systematisierender Herangehensweise erhält der Leser hier weiterführende Einsichten im Sinne einer fraktionsorientierten Parlamentarismusforschung.

Helge F. Jani

\title{
Parteien in Thüringen: Meilenstein der regionalen Parteienforschung
}

\author{
Schmitt, Karl und Torsten Oppelland (Hrsg.): Parteien in Thüringen. Ein Handbuch, Droste \\ Verlag, Düsseldorf 2008, 628 Seiten, $€ 78$,-.
}

Die Erweiterung des bundesdeutschen Parteiensystems nach Osten im Zuge der deutschen Einigung zählt zu jenen Teilen des Institutionentransfers, die zwar nicht gänzlich ohne innere Friktionen, aber im Ergebnis doch zügig und weitgehend geräuschlos über die historische Bühne gegangen sind. Im Ergebnis blieb das in Westdeutschland gewachsene Parteiensystem in seinem Grundmuster unverändert, wenngleich zunächst ergänzt um eine linkssozialistische ostdeutsche regionale Interessenpartei in Gestalt der PDS, der erst ab 2007, nach der Fusion mit der WASG zur Partei Die Linke, die Westausdehnung gelang. Zur raschen Verschmelzung trug zum einen wesentlich mit bei, dass politisch interessierte Ostdeutsche schon lange vor der Einigung eine „Quasi-Parteiidentifikation“" (Carsten Bluck I Henry Kreikenbohm) mit einer präferierten Westpartei aufgebaut hatten. Zum anderen fand Ostdeutschland im Wählerverhalten auch wieder Anschluss an die alten Konfliktlinien („Cleavages“). Bei genauerem Hinsehen werden indes auch ostdeutsche Eigenheiten sichtbar, die in das gesamtdeutsche Parteiensystem dauerhaft Eingang gefunden haben. So hat sich das seit 1990 existierende West-Ost-Gefälle in der Zahl der Parteimitglieder eher noch verstärkt. Die Bindung an ihre jeweilige Partei fällt bei ostdeutschen Parteimitgliedern schwächer aus. Die Abneigung, Fraktionsdisziplin zu üben, ist unter Parlamentariern im Osten stärker ausgeprägt. Und auf der Links-Rechts-Achse, die zur Messlatte politischer Grundüberzeugungen immer noch taugt, ordnen sich Mitglieder ostdeutscher Landesparteien im Mittel näher zum linken Pol ein als westdeutsche Gesinnungsfreunde.

Solche ostdeutschen Besonderheiten im Parteiengefüge, die fortbestanden beziehungsweise sich nach 1989/90 deutlicher ausformten, erschließen sich dem aufmerksamen 
Betrachter genauer auf der regionalen Ebene. Der Verfassungsstruktur und politischen Handlungslogik des Föderalstaates folgend, haben sich auch im Osten der Republik eigenständige Landesparteien ausgebildet, die die nach dem Ende der DDR wieder erkennbare Bandbreite historisch gewachsener Traditionszonen und regionaler politischer Kulturen widerspiegeln. So ist es denn auch nicht nur ein dem Landesbewusstsein förderliches, sondern auch wissenschaftlich wohlbegründetes Unterfangen, den Werdegang und aktuellen Entwicklungsstand der Parteiensysteme der neuen ostdeutschen Länder gesondert aufzuarbeiten.

Für diese regionale Spezifikation der Parteienforschung ist das von den Jenaer Politikwissenschaftlern Karl Schmitt und Torsten Oppelland herausgegebene Handbuch zu den Parteien Thüringens im besten Sinne beispielhaft. Diese erste umfassende Darstellung von Parteien und Parteiensystem in Thüringen umschließt zeitlich fast zwei Jahrzehnte seit der Phase der Formierung (1989/90) des neuen demokratischen Parteienspektrums. Die Parlamentsparteien CDU (Autor: Thomas Sauer), PDS (Thomas Sauer / Torsten Oppelland), SPD (Matthias Bettenhäuser / Sebastian Lasch), FDP (Andreas Hallermann) und Bündnis 90/Die Grünen (Sven Leunig / Björn Memmeler) werden in ausführlichen Teilkapiteln jeweils monographisch abgehandelt. Ein weiteres Kapitel befasst sich mit den rechtsextremen Parteien DVU, NPD und REP (Janine Patz / Torsten Oppelland), die seit 1990 nicht im Erfurter Landtag vertreten sind (ein Thüringer Alleinstellungsmerkmal im ostdeutschen Landesparlamentarismus).

Ein umfangreicher Anhang dokumentiert auf gut 90 Seiten kurze Chroniken der Parteigründungen, Wahlergebnisse für Landtags und Kommunalwahlen, die Verteilung der Mandate, die Zusammensetzung der Landeskabinette und Kurzbiographien führender Landespolitiker.

Die Parteien-Kapitel folgen einem einheitlichen Gliederungsmuster: Einem historischen Abriss, der die Vorgeschichte in der defekten demokratischen Interimsphase zwischen Kriegsende 1945 und der Gründung der DDR 1949 beziehungsweise der Länderauflösung 1952 beleuchtet, schließen sich Abschnitte an zur Formierung der neuen (beziehungsweise erneuerten) Partei während der Umbruchzeit 1989/90, zu Programmatik und Strategie, zur Organisation (vertikale und subregionale Gliederung, Vereinigungen, Finanzen, Parteimedien), zur zahlenmäßigen Größe, sozialen Zusammensetzung und territorialen Verteilung der Mitglieder, zur Anatomie der Wähler, zur Vernetzung im vorpolitischen Raum und zu künftigen Entwicklungsperspektiven. Die Handlungsebenen der „Partei im Parlament“ und der „Partei in der Regierung“ (oder Opposition) werden nicht, wie man zunächst erwarten mag, in separat ausgewiesenen Teilabschnitten untersucht, aber in andere Passagen integriert und somit nicht vernachlässigt.

Die Parteien-Kapitel bestechen ausnahmslos durch minutiös aus Primärquellen und Sekundärliteratur rekonstruierte Chroniken der Formierungs- und Konsolidierungsphase (die etwa 1991 abgeschlossen ist), ferner durch eine profunde Detailkenntnis (die niemals den roten Pfaden der Darstellung verloren gehen lässt) sowie eine abwägende Urteilskraft (die sich über Legendenbildung beteiligter Zeitzeugen und parteioffizielle Lyrik souverän erhebt). Der Qualität des Buches kommt erkennbar zugute, dass es aus einem DFG-geförderten Forschungsprojekt hervorgegangen ist. Die in diesem Kontext durchgeführte repräsentative Mitgliederbefragung in den Landesparteien (1997/98) bietet eine empirisch fundierte und bisher einzigartige Grundlage, um beispielsweise eine nach einheitlichen Kategorien erhobene Sozialdemographie der Mitgliedschaften zu präsentieren. 
Die Zusammenführung der einzelnen Landesparteien und ihrer regionalen Ontogenese in der Arena jener Beziehungen, die ein Parteiensystem ausmachen, erfolgt in einem einführenden und einem schließenden Kapitel. Einführend zeichnet Karl Schmitt die "große Wegstrecke von der Parteidiktatur zur Parteiendemokratie" in Thüringen nach. Die Neuformierung der Parteienlandschaft stand zu Beginn im Zeichen nicht nur der „Reföderalisierung der DDR", sondern auch noch jener historisch gewachsenen, klassischen Konfliktlinien, die der SED-Staat bemerkenswerterweise nicht völlig hatte zerstäuben können. „Die Vorstellung, der DDR sei 1990 ein völlig fremdes Parteiensystem übergestülpt worden, greift also zu kurz" (S. 25). Ähnlich der Zeit kurz nach der Demokratiegründung in Westdeutschland 1945/49, traten auch in Thüringen 1989/90 alte Kontinuitäten der Lagerbindung und politisch-kulturellen Orientierung wieder zutage. Die hergebrachten Konfliktlinien Kirche versus Staat und des Klassengegensatzes übertrugen sich abermals in das Wahlverhalten, jedoch im Zeichen einer „neue[n] Zuordnung von Sozialstruktur und Parteiensystem" (S. 36): Arbeiter und kirchlich gebundene Bevölkerungsteile wandten sich der CDU zu, SED-Kader und Personengruppen, die zu DDR-Zeiten systemloyal gewesen waren, der PDS. Liberale und SPD, das zeichnete sich in Thüringen bereits früh ab, waren die Verlierer im Prozess dieser elektoralen Polarisierung.

Im Schlusskapitel (Karl Schmitt / Torsten Oppelland) wird die Frage „Gelungene Konsolidierung?" mit Rückgriff auf weitere empirische Daten der Mitgliederumfrage aufgenommen. Für die dauerhafte Festigung der Parteiorganisationen sind Menge, Motivation und Partizipation der Mitglieder elementare Voraussetzungen. Im Spiegel der Daten können die Verfasser zeigen, dass die Aktivitätsprofile variieren, teils mit dem Alter der Mitglieder (wie vor allem bei der PDS), aber auch mit der Gesamtmitgliederzahl und der Anzahl zu besetzender Mandate. Quer über alle Landesparteien hinweg ist ein langfristig rückläufiger Trend bei den Mitgliederzahlen zu beobachten. Hinsichtlich der Erosion der Mitgliederpartei ist Thüringen keine Ausnahme. Zu Recht machen die Verfasser diesen für die $\mathrm{Zu}-$ kunft des Parteienstaates kritischen Punkt zur Botschaft ihrer Schlussformel: „Auch hier gilt, dass den Parteien, je weniger Mitglieder sie haben, ihre Rekrutierungsbasis für qualifiziertes politisches Führungspersonal ebenso verloren geht wie die lebendige Verbindung zu den Interessen und Problemen der Bürger" (S. 493).

Das vorliegende Thüringer Parteienhandbuch stellt einen Meilenstein der regionalen Parteienforschung dar, der für Folgepublikationen weiterer Bundesländer, in Ost- wie Westdeutschland, hohe Maßstäbe setzt.

Everhard Holtmann

\section{Linke und rechte Kleinparteien: wenig Neues}

Bolay, Raphael: Erfolgreiche Linke, gescheiterte Rechte. Ursachen für die Asymmetrie des deutschen Parteiensystems, Tectum Verlag, Freiburg 2010, 135 Seiten, € 24,90.

Klassische rechtspopulistische Parteien konnten - im Unterschied zur westeuropäischen Erfolgswelle - in Deutschland landesweit kaum reüssieren und niemals in den Bundestag einziehen. Der kurzfristige Erfolg eines Ronald B. Schill im Stadtstaat Hamburg liegt bereits fast ein Jahrzehnt zurück. Im Unterschied dazu gelang es der PDS, in Ostdeutschland min- 